\title{
Comparison of shutter glasses and mirror stereoscope for measuring dynamic and static vergence
}

\author{
Wolfgang Jaschinski \\ University of Dortmund, Institut für Arbeitsphysiologie, Germany \\ Stephanie Jainta \\ University of Dortmund, Germany \\ Jörg Hoormann \\ University of Dortmund, Germany
}

\begin{abstract}
Vergence eye movement recordings in response to disparity step stimuli require to present different stimuli to the two eyes. The traditional method is a mirror stereoscope. Shutter glasses are more convenient, but have disadvantages as limited repetition rate, residual cross task, and reduced luminance. Therefore, we compared both techniques measuring (1) dynamic disparity step responses for stimuli of 1 and 3 deg and (2) fixation disparity, the static vergence error.

Shutter glasses and mirror stereoscope gave very similar dynamic responses with correlations of about 0.95 for the objectively measured vergence velocity and for the response amplitude reached $400 \mathrm{~ms}$ after the step stimulus (measured objectively with eye movement recordings and subjectively with dichoptic nonius lines). Both techniques also provided similar amounts of fixation disparity, tested with dichoptic nonius lines.
\end{abstract}

Keywords: vergence eye movements, disparity vergence, fixation disparity, dichoptic separation

\section{Introduction}

When we change the point of regard between objects at different viewing distances in natural vision, vergence eye movements are required to project the point of regard onto the centre of the fovea in each eye. In the ideal case, the visual axes intersect at the target point and form a vergence angle that depends on the viewing distance and the inter-pupillary distance. In laboratory conditions, the properties of the vergence system have widely been investigated with stimuli at a fixed viewing distance, but with a horizontal displacement of the stimuli presented to the two eyes, in order to induce a retinal disparity between the left and right eye (Howard, 2002). Such a dichoptic presentation can - for example - be realized with a mirror stereoscope or with electronic shutter glasses. It is the aim of the present methodological study to compare these two methods in order to see whether similar vergence responses might be induced by these two instru- mentations.

At a mirror stereoscope, the separate images (on two displays or on smaller areas on one display) are combined with mirrors at right angle. This traditional method of dichoptic presentation (following Wheatstone) has the disadvantage that surface mirrors are required at precisely adjusted positions relative to the eyes and displays. A mechanically more easy method are shutter glasses and cathode ray tubes (CRT) under computer control: the series of consecutive frames on the CRT screen is presented alternately to the left and right eye by switching the optical transmission of the shutter glasses between closed and transparent; this is made synchronously with the refresh rate of the CRT screen. Although shutter glasses are very convenient to use, they have several potential disadvantages.

(1) Even if the CRT is run at a high refresh rate of, e.g., $120 \mathrm{~Hz}$, the refresh rate for the single eye is only half as high, thus the display may not be free of flicker. 
(2) The images are not presented simultaneously, but alternating to the left and right eye, i.e. when one eye is viewing the target, the fellow eyes is covered.

(3) The alternating covering of the eyes is not perfect, thus, each eye may have a faint perception of the image intended to be visible only by the fellow eye. In order to eliminate this cross-talk between the eyes, in the present study, we used a reduced contrast of the stimuli on a bright background, which attenuates the cross-talk below threshold. (4) The resulting luminance of the stimuli is reduced due to the alternating covering of the eyes and the attenuation even in the transparant state of the shutter glasses. It is unclear whether these various limitations of the shutter technique may have an effect on the physiological measures to be investigated. Therefore, we made comparative measurements with a mirror stereoscope where the limitations of the shutter technique do not apply.

As physiological measures we investigated disparityinduced vergence step responses and fixation disparity, i.e. the vergence error when a stationary fusion stimulus is presented. It is known from previous research and from optometry that subjects with normal binocular vision differ considerably in these vergence parameters (e.g., Jones, 1977; Jainta et al., 2007; Jaschinski et al., 2008). In order to see whether these individual differences are reflected by the shutter glasses and the mirror stereoscope, we performed regression analyses between results of both techniques.

In Experiment 1, we presented disparity step stimuli of 1 and 3 deg amplitude (in the convergent and divergent direction) and measured the dynamic vergence responses simultaneously with objective recordings and a subjective technique using two dichoptically presented nonius lines (one presented to each eye). In Experiment 2, we used dichoptic nonius lines for measuring fixation disparity (Howard, 2002). In a concurrent control condition, nondichoptically presented nonius lines were applied for measuring the nonius bias, i.e. the physical horizontal nonius offset required for perceived alignment (Jaschinski et al., 1999).

\section{Methodology}

The methods applied in Experiment 1 and Experiment 2 have been reported in Jainta et al. (2007) and Jaschinski et al. (2004), respectively. The conditions of the present experiments were as follows. The viewing distance was $60 \mathrm{~cm}$ in all conditions.

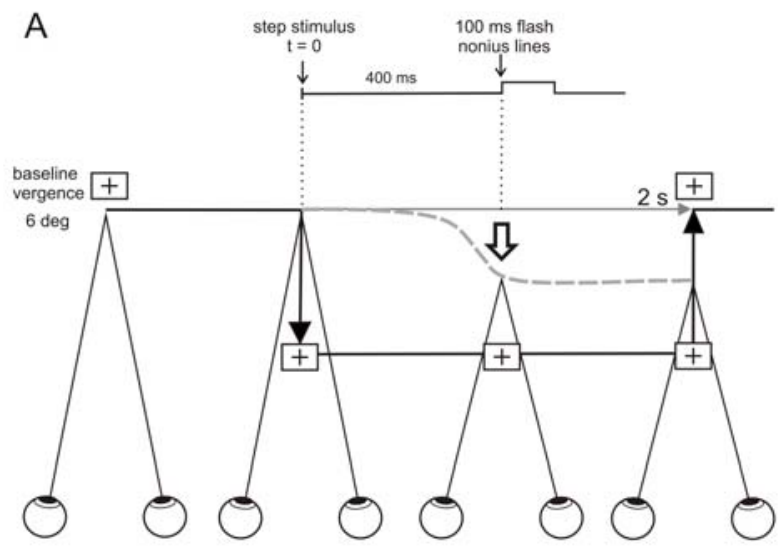

B

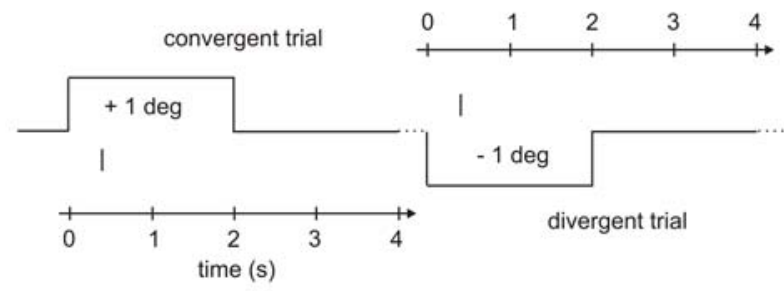

Figure 1. (A) In the beginning of a trial, the stimulus (illustrated by the central fixation cross surrounded by a rectangular frame, not to scale) is presented with zero disparity (i.e. at a baseline vergence angle of about 6 deg). At $t=0$, the step stimulus of disparity (convergent in this example) is presented and the eyes perform a convergent movement, schematically illustrated by the dashed line. With a delay of $400 \mathrm{~ms}$, nonius lines are flashed (for $100 \mathrm{~ms}$ ) in order to estimate the vergence state reached within $400 \mathrm{~ms}$ (indicated by the open arrow). At $2 \mathrm{~s}$ after onset of the convergent step stimulus, the baseline vergence stimulus appears again. (B) A sequence of a convergent and a divergent trial with a disparity step stimulus of 1 deg. The tick on the time axis indicates the moment when the nonius lines were flashed. 20 convergent and 20 divergent trials were randomly interleaved in order to run two psychometric procedures, one for each vergence direction. 
In Experiment 1, the fixation stimulus contained a black frame (6.2 deg wide x 4.3 deg high; 0.3 deg stroke width) with a central fixation cross ( 0.5 deg; stroke width $0.1 \mathrm{deg})$. The monocular nonius lines for the right and left eye were presented above and below the fixation cross; the vertical nonius lines were 1 deg high ( 0.3 deg stroke width) and had a vertical separation of $0.8 \mathrm{deg}$. Each test comprised a series of vergence responses elicited by introducing disparity step stimuli (20 trials in the convergent and divergent direction, respectively, that were randomly interleaved). Separate tests were made with step stimuli of 1 deg and $3 \mathrm{deg}$. For measuring the vergence response subjectively, dichoptic nonius lines (the upper line was only visible by the right eye and the lower line only by the left eye) were flashed for 100 ms with an onset-delay of $400 \mathrm{~ms}$ after the step stimulus (see Figure 1). The delay of $400 \mathrm{~ms}$ was chosen to be longer than the latency of about $200 \mathrm{~ms}$ and shorter than the time required to fully complete the vergence movement which can be about $1 \mathrm{~s}$ for small stimulus amplitudes; the resulting measure describes the initial vergence response which is predominantly open-loop and little affected by feedback control (Howard, 2002). During a test, the amount of the horizontal nonius offset was varied in a series of 20 trials according to a psychometric procedure that automatically tracks the point of equality, where the upper line is perceived to the right or to the left of the lower line with 50\% probability (Best PEST; Lieberman \& Pentland, 1982). This procedure determines the physical nonius offset corresponding to perceived alignment in order to estimate the amount of the dynamic vergence response (or fixation disparity in Experiment 2). For comparison, vergence eye movements were simultaneously recorded objectively with an EyeLink II system at $500 \mathrm{~Hz}$ sampling rate, which gave objective measures of the maximal vergence velocity and the vergence state $400 \mathrm{~ms}$ after the disparity stimulus onset (for details of data analyses, see Jainta et al., 2007). The EyeLink II is specified to have a resolution of $0.01 \mathrm{deg}=0.6 \mathrm{~min}$ arc. This technical specification shows that the EyeLink II is useful for our recordings. The quality of the actual measurements further depends on the stability of recording conditions. The observed high correlations between mirror stereoscope and shutter glasses confirm that our recordings and procedures gave reliable results. We made two repeated tests in separate sessions and averaged the individual results for each of 8 subjects.
In Experiment 2, the stationary fusion stimulus included a centre string of the three letters XOX (2.7 deg wide and 0.6 deg high, 0.2 deg stroke width), relative to which an upper and a lower nonius line was presented (1 deg single line length, 1.4 deg central separation, 0.1 deg stroke width); additionally, a stationary peripheral frame (6.2 deg wide and 4.3 deg high, 0.3 deg stroke width) assisted fusion. Two psychometric tests (Best PEST) were run for measuring concurrently the fixation disparity and the nonius bias. For the fixation disparity test, the nonius lines were presented dichoptically. For the nonius bias test, the nonius lines were presented nondichoptically (upper and lower line both visible for the right and left eye). In each test, a series of 50 flashes (100 ms) of the nonius lines were used, randomly interleaved for both tests. 12 subjects were tested in two separate sessions that each included four repeated tests in order to average across intra-individual variability.

In the mirror stereoscope, two surface mirrors were placed at right angle and let the subjects view the targets for the right and left eye on two CRT monitors placed at the right and left side. The two CRT screens were operated in a way that the series of 100 frames per second were presented simultaneously to the two eyes. The black stimulus element were presented on bright background (luminance $26 \mathrm{~cd} / \mathrm{m}^{2}$ with $100 \%$ contrast).

The shutter glasses (Elsa revelator), were operated at $120 \mathrm{~Hz}$, thus each eye had a repetition rate of $60 \mathrm{~Hz}$, i.e. the stimuli for the right and left eye were interleaved by one frame cycle. The bright background luminance (as viewed through the shutter glasses) was $10 \mathrm{~cd} / \mathrm{m}^{2}$ and the stimulus elements were not black, but had $4 \mathrm{~cd} / \mathrm{m}^{2}$ luminance in order to eliminate visible cross-task between the eyes (see Introduction).

Subjects had good stereoscopic vision tested with the TNO test; partly, they wore refractive corrections in order to have visual acuity of 1.0 or better in each eye. Subjects were instructed to fixate the centre fixation target; they responded with the computer mouse whether the upper nonius line was perceived left or right relative to the lower nonius line.

\section{Results}

Figure 2 shows the results of the individual mean vergence step responses described by three measures of Experiment 1: the maximal vergence velocity (measured objectively) and the amount of the response at the mo- 
ment in time $400 \mathrm{~ms}$ after the disparity step stimulus; the latter was measured objectively and subjectively (with nonius lines). We plotted regression lines for each measure between the results of the mirror stereoscope and the shutter glasses in order to test whether the individual differences in vergence responses are reflected by both methods. Each graph in Figure 2 includes the results of convergent and divergent step responses, separate plots are shown for step stimuli of $1 \mathrm{deg}$ and $3 \mathrm{deg}$. For each condition, the regression line did not differ significantly from the identity line, since the y-intercept did not differ significantly from 0.0 and the slope did not differ signifi- cantly from 1.0. The correlation coefficients ranged between 0.87 and 0.98 .

If we consider the response reached $400 \mathrm{~ms}$ after the step stimulus for the 3 deg disparity steps, we found that about 4 of the 8 subjects had responses less than 60 min arc, i.e. less than $1 / 3$ of the step stimulus of 180 min arc. Such relatively small responses were not found for the 1 deg stimulus, where nearly all responses exceeded $20 \mathrm{~min}$ $(1 / 3$ of $60 \mathrm{~min}$ arc $=1 \mathrm{deg})$.

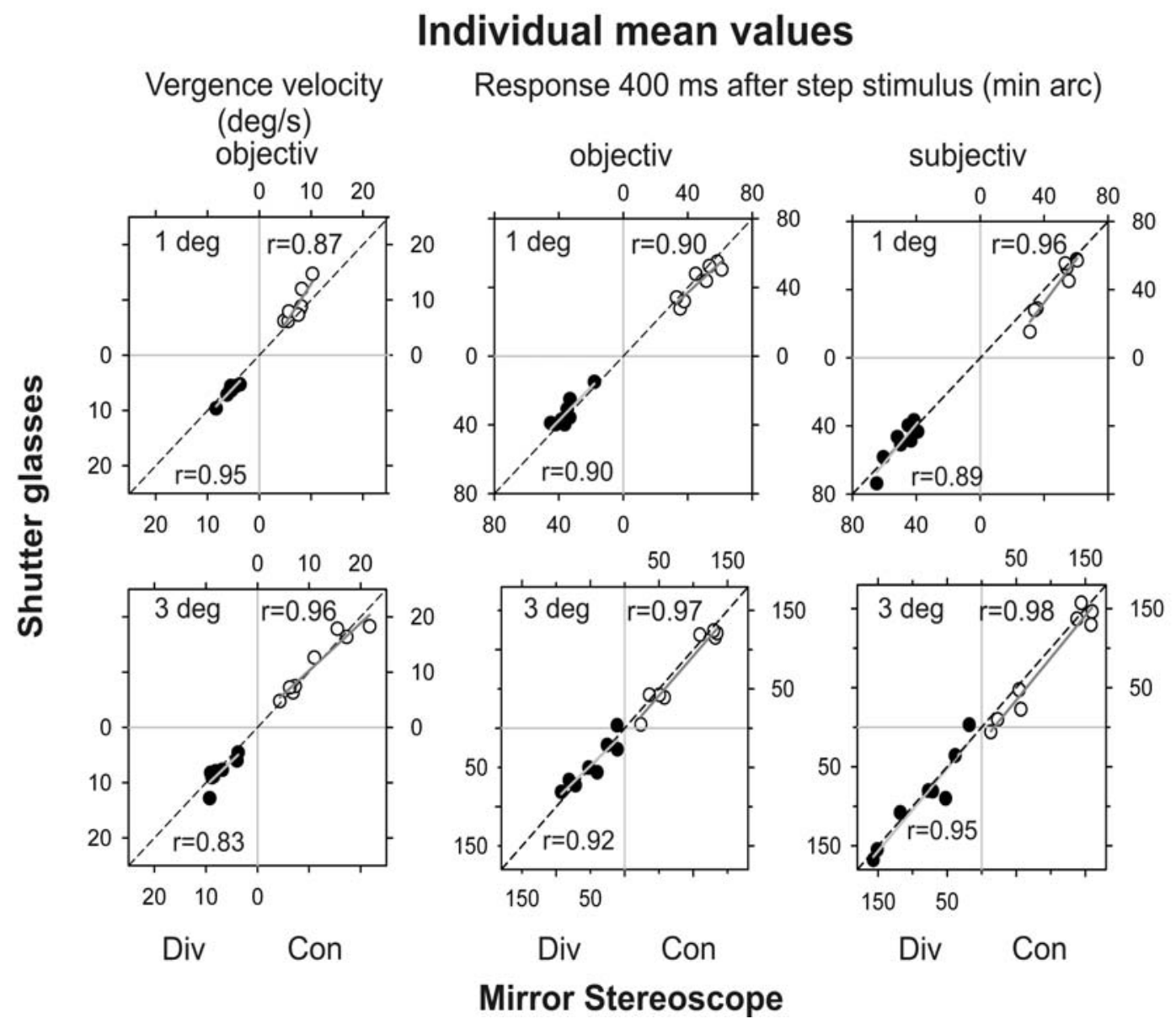

Figure 2. Each graph gives the comparison of shutter glasses and mirror stereoscope for the individual mean values of 8 subjects. The three dependent measures are the objectively measured maximal vergence velocity and the response reached at a moment in time 400 ms after the disparity step stimulus, measured objectively and subjectively (with nonius lines). Upper and lower graphs refer to the 1 deg and 3 deg disparity step stimulus. Convergent responses are shown by open symbols in the upper right quadrants, divergent responses by closed symbols in the lower left quadrants.. Note that for each graph, regression lines and Pearson correlation coefficients are given separately for convergent and for divergent step responses. The identity line is shown as a reference. 


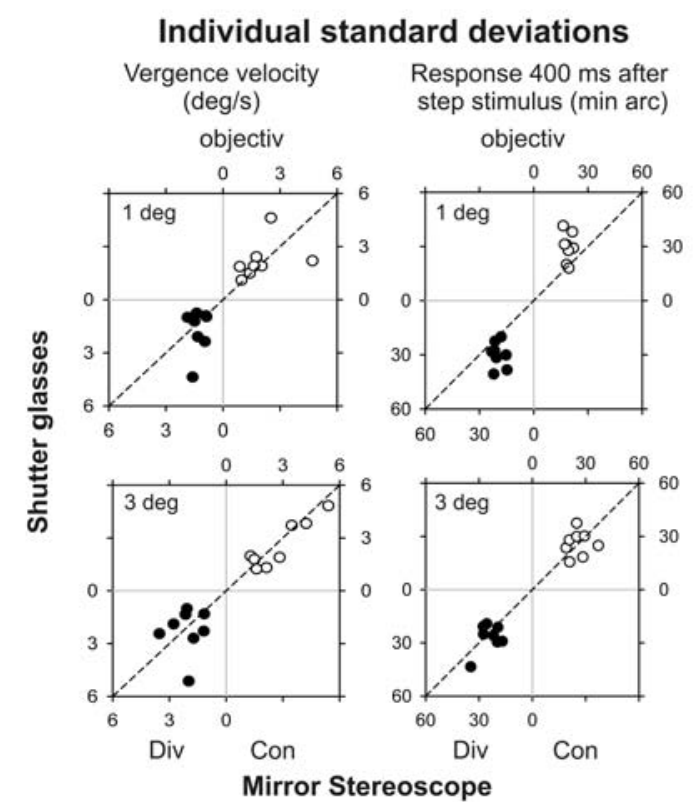

Figure 3. Each graph gives a comparison of shutter glasses and mirror stereoscope for the standard deviations of 8 subjects. These data refer to the objective measures, i.e the maximal vergence velocity and the response reached at a moment in time $400 \mathrm{~ms}$ after the disparity step stimulus. Convergent responses are shown by open symbols in the upper right quadrants, divergent responses by the closed symbols in the lower left quadrants. Upper and lower graphs refer to the 1 deg and 3 deg disparity step stimulus. The identity line is shown as a reference.

The response reached $400 \mathrm{~ms}$ after the disparity step stimulus was measured simultaneously with the objective and the subjective method. In the present experimental design we had 8 comparisons of objective and subjective measures that gave correlation coefficients ranging between 0.45 and 0.98 (median 0.90).

In order to describe the variability within subjects, the standard deviations across all available trials for each subject are plotted in Figure 3 for the objective measures; up to 20 trials per session were available for each condition. For most conditions, the individual standard deviations are scattered around the identity line, thus they were similar with both techniques. Only for the response $400 \mathrm{~ms}$ after the step stimulus of 1 deg, the intraindividual standard deviations tended to be larger and to scatter more strongly among subjects, if the shutter technique was used as compared to the mirror stereoscope. We confined this analysis of intra-individual variability to the description in Figure 3, since regressions or tests of significance are questionable based on standard deviations. Intra-individual standard deviations cannot be provided for the subjective measure of the response $400 \mathrm{~ms}$ after the step stimulus (Figure 2, right graphs), since the series of 20 left/right-responses in the psychometric test procedure allows for an individually reliable estimation of mean values, but not of variability.

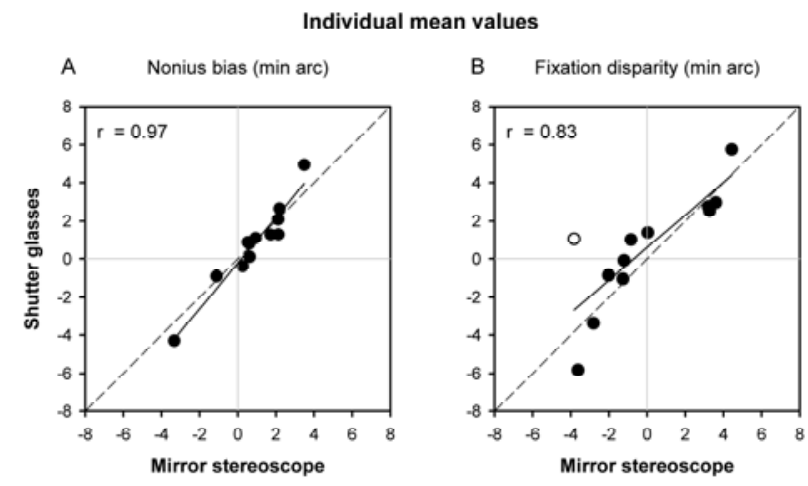

Figure 4. Comparison of shutter glasses and mirror stereoscope for the individual mean values of 12 subjects for the nonius bias (A) and the fixation disparity (B). The Pearson correlation coefficient is given and the regression line is included; the identity line is shown as a reference. In fixation disparity, positive (negative) figures represent over-convergence (underconvergence) relative to accurate vergence angle (zero fixation disparity). In nonius bias, positive (negative) figures represent that the upper line has to be shifted physically to the left (right) of the lower line for perceived alignment.

The individual mean values of the tests with the stationary vergence stimuli are shown in Figure 4. The nonius bias was very similar with the mirror stereoscope and the shutter glasses as shown by the high correlation of $\mathrm{r}=0.96$ and a regression line that did not differ significantly from the identity line (Figure 4A). Thus, the judgment of nonius lines relative to each other was performed in the same way for both techniques of presentation, which is a prerequisite for finding the same fixation disparity. For the fixation disparity (Figure 4B), most data were very similar in both conditions and the regression line did not differ significantly from the identity line. However, the correlation coefficient $(r=0.83)$ was smaller than for the other measures, because one subject (shown by the open symbol) had a rather large and individually significant $(\mathrm{p}<0.0001)$ difference between the two meth- 
ods $(1.0 \pm 2.1$ versus $-3.8 \pm 2.2 \mathrm{~min}$ arc $)$. This subject reported after the test, that she adopted different strategies of viewing in the two conditions: with the shutter glasses she viewed at the screen as usually during computer work since the distance to the screen was directly perceived. However, this was not possible at the mirror stereoscope, so that she described to view at distance into free space (as she did during her juggling exercises, she reported). Thus, the imagination of viewing at distance appeared to shift her fixation disparity by $4.8 \mathrm{~min}$ arc into the under-convergent direction. For this subject, the nonius bias gave very similar results with the mirror stereoscope and the shutter glasses $(0.3 \pm 0.8$ and $-0.4 \pm$ $1.1 \mathrm{~min}$ arc, respectively), so that we conclude that the difference in fixation disparity is not a result of the performance in nonius judgment, but due to different vergence states. Without this single subject (open symbol), the correlation for fixation disparity increased to $r=0.92$.

For the subjective measures of nonius bias and fixation disparity, standard deviations corresponding to the individual mean values in Figure 4 can be calculated across the 8 repeated tests per subject. These standard deviations are shown in Figure 5 in order to compare shutter glasses and mirror stereoscope. The scatter plots suggest similar intra-individual variability for the two techniques.
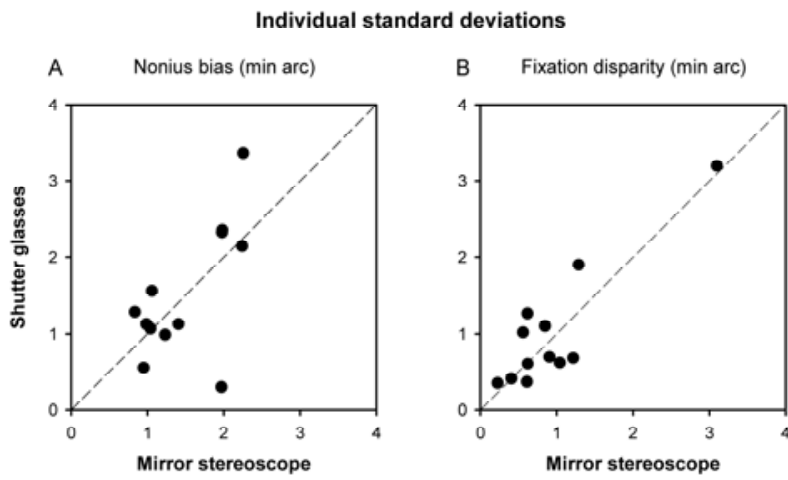

Figure 5. Comparison of shutter glasses and mirror stereoscope for the intra-individual standard deviations of 12 subjects for the nonius bias (A) and the fixation disparity (B).

\section{Discussion}

As in previous studies with non-selected samples of subjects with normal binocular vision (e.g., Jones, 1977; Jainta et al., 2007; Jaschinski et al., 2008) we found that our participants differed considerably in the vergence measures investigated. The responses to the disparity vergence step stimuli of 1 and 3 deg revealed that some subjects had relatively small, and even missing responses at the larger 3 deg stimulus; this observation resembles the finding of Jones (1977) that an increase in stimulus can lead to a reduction in response.

We did not select our participants with respect to optimal performance since the variability in vergence performance among subjects may be relevant for practical optometric testing of binocular vision. In this respect it is relevant to know whether shutter glasses - the technically more easy method for dichoptic separation - is able to reflect the individual differences in the same way as the traditional mirror stereoscope.

Although the viewing conditions with shutter glasses differ in some respect from those in a mirror stereoscope (see Introduction), the physiological functions investigated in the present study were very similar with both techniques on the level of individual mean values. Thus, the putative disadvantages of shutter glasses do not apply for measuring dynamic disparity vergence responses, both in objective and subjective methods for vergence testing. For a stationary vergence stimulus, the nonius bias and the fixation disparity was also equivalent in most subjects. Only one subject showed an under-converging fixation disparity due to an disturbing proximal vergence effect at the mirror stereoscope where one is not directly aware of the actual viewing distance (Hung et al., 1996). In this respect, shutter glasses allow for more natural viewing conditions.

The intra-individual standard deviations described the variability from trial to trial for the objective measures of vergence step responses and the variability from test to test for the subjective measures of nonius bias and fixation disparity. Generally, individual standard deviations were similar between shutter glasses and mirror stereoscope. Only for 1 deg step stimuli, the response $400 \mathrm{~ms}$ after the step stimulus tended to have more variability with shutter glasses, thus averaging across a series of trials is advisable. 
The high correlation between the objective and the subjective technique for measuring the response $400 \mathrm{~ms}$ after the step stimulus confirms that also the subjective nonius technique is able to detect whether a subject has a relatively small or large vergence step response. This issue was specifically addressed in the study of Jainta et al. (2007).

In conclusion, as it was expected from previous studies with large, non-selected samples of subjects with normal binocular vision, we replicated considerable individual differences between our subjects in their disparityinduced vergence step responses, nonius bias and fixation disparity. The high correlations between the individual mean results of mirror stereoscope and shutter glasses show that the technically more simple shutter glasses are able to reflect these individual differences.

\section{Acknowledgement}

The authors wish to thank Claudia Haensel and Michael Bachseitz for testing the subjects.

\section{References}

Howard, I.P. (2002). Seeing in Depth; Basic Mechanisms. 1. Toronto, Canada: Porteous, I.

Hung, G.K., Ciuffreda, K.J., \& Rosenfield, M. (1996). Proximal contribution to a linear static model of accommodation and vergence. Ophthalmic and Physiological Optics, 16, 31-41.

Jainta, S., Hoormann, J., \& Jaschinski, W. (2007). Objective and subjective measures of vergence step responses. Vision Research, 47, 3238-3246.

Jaschinski, W. (2004). Relation between static and dynamic aspects of vergence, estimated with a subjective test using flashed dichoptic nonius lines. Ophthalmic and Physiological Optics, 24, 399-410.

Jaschinski, W., Bröde, P., \& Griefahn, B. (1999). Fixation disparity and nonius bias. Vision Research, 39, 669-677.

Jaschinski, W., Švede, A., \& Jainta, S. (2008). Relation between fixation disparity and the asymmetry between convergent and divergent disparity step responses. Vision Research, 48, 253-263.
Jones, R. (1977). Anomalies of disparity detection in the human visual system. Journal of Physiology, 264 (3), 621-640.

Lieberman, H.R. \& Pentland, A.P. (1982). Microcomputer-based estimation of psychophysical thresholds: The Best-PEST. Behaviour Research Methods and Instrumentation, 14, 21-25. 